J. Japanese Soc. Comp. Statist. 6.2(1993), 1-10

\title{
ESTIMATE OF VARIANCE OF WILCOXON-MANN-WHITNEY STATISTIC
}

\section{S. SHIRAHATA*}

\begin{abstract}
The problem of estimating the variance of the Wilcoxon-Mann-Whitney statistic is considered. Minimum variance unbiased estimator which is a $U$-statistic, bootstrap estimator, an estimator proposed by Fligner \& Policello(1981) and jackknife estimator are considered. We also consider induced estimators of the standard deviation and interval estimates of the expectation of the Wilcoxon-Mann-Whitney statistic. By computer simulations, we conclude that bootstrap estimator is efficient for both the variance and the standard deviation in the sense of mean squared error. This is the same conclusion with Sakamoto \& Shirahata(1992) where the estimating problem of variance of one-sample $U$-statistics is considered. It is, in addition to the above, found that the mean squared error of minimum variance unbiased estimator is small and the bias of the induced estimator is also small for the standard deviation. Fligner \& Policello estimator gives accurate confidence coefficients for many cases and the jackknife estimator stands between the former two estimators and Filgner \& Policello estimator.
\end{abstract}

\section{Introduction}

Let $X_{1}, \ldots, X_{m}$ and $Y_{1}, \ldots, Y_{n}$ be random samples from continuous distribution functions $F(x)$ and $G(y)$, respectively. Consider the Wilcoxon statistic

$$
W=\sum_{i=1}^{m} R_{i}
$$

given by Wilcoxon(1945) where $R_{i}$ is the rank of $X_{i}$ among the combined sample. The statistic $W$ is equivalent to

$$
U=U_{m n}=\frac{1}{m n} \sum_{i=1}^{m} \sum_{j=1}^{n} u\left(X_{i}-Y_{j}\right)
$$

${ }^{*}$ College of General Education, Osaka University Key words: Bootstrap; Estimate of standard deviation; Estimate of variance; Fligner \& Policello estimator; Interval estimate; Jackknife; Mann-Whitney; Unbiased; Wilcoxon. 


\section{SHIRAHATA}

which is given by Mann \& Whitney(1947) where $u(x)=1$ or 0 according as $x \geq 0$ or $x<0$. The statistic $U$ is more convenient for us and we call $U$ Wilcoxon-Mann-Whitney statistic. The statistic $U$ is widely applied to test the null hypothesis that the medians of $F$ and $G$ are identical with each other. However, from the definition of $U$, the null hypothesis should be $H: \theta=\frac{1}{2}$ where $\theta=P(X>Y)$. The critical points of the tests based on $U$ by the normal approximation

$$
U \approx N\left(\frac{1}{2}, \frac{m+n+1}{12 m n}\right)
$$

or exact percentage points given in, for example, Milton(1964) are usually applied. The approximation (3) and the exact points are true only when the hypothesis $H^{\prime}: F(x) \equiv G(x)$ holds. In general, it is not correct to use (3). Furthermore, the statistic $U$ induces a nonparametric estimator (Hodges-Lehmann estimator given in Hodges \& Lehmann(1963) and Lehmann(1963)) of $\xi$ under the model

$$
G(x)=F(x+\xi) .
$$

The confidence limits of the estimate is also selected from the approximation (3). The model (4) implies that $F(x)$ and $G(x)$ are different only in their location parameters. In practice, it is difficult to check whether the model holds or not. Hence, in the problem of interval estimating the difference between $F$ and $G$, we should estimate $\theta$.

In this paper, we consider the problem of estimating the variance $\operatorname{var}(U)=\sigma^{2}=\sigma_{m n}^{2}$ and the standard deviation $\sigma$. Since the asymptotic normality

$$
U \approx N\left(\theta, \sigma^{2}\right)
$$

holds for large $m$ and $n$, we can test the hypothesis $H$ and give an interval estimator of the parameter $\theta$ asymptotically, provided $\sigma^{2}$ is properly estimated.

As estimators of $\sigma^{2}$, we consider the minimum variance unbiased estimator (which is a $U$-statistic and we call it U-estimator), bootstrap estimator (B-estimator), an estimator given in Fligner \& Policello(1981)(F-estimator) and jackknife estimator (J-estimator). We can calculate the mean squared errors of these four estimators for a specified population distribution. However, the task requires a formidable amount of calculations. Hence the comparisons are performed by computer simulations. We also compare the squared roots of the estimators as estimators of the standard deviation $\sigma$. We also call the induced estimators U-, B-, F-, and J-estimator. It is shown that the B-estimator is the best in the sense of mean squared error for both $\sigma^{2}$ and $\sigma$ and the bias of the U-estimator is also small for $\sigma$. In Sakamoto \& Shirahata(1992), estimators of the variance of several $U$-statistics were considered and it is concluded that Bootstrap estimator is the best and the accuracy of the best unbiased estimator is not so inferior to the Bootstrap estimator. The results of this paper are similar to that of Sakamoto \& Shirahata(1992).

We further compare the interval estimators of $\theta=P(X>Y)$. The interval estimators are given by $U$ standardized by our estimators of $\sigma$. The confidence limits are given by normal or $t$-approximations with or without finite corrections. It is shown that the $\mathrm{F}$ estimator gives accurate confidence coefficients in many cases though it is inferior to the $\mathrm{U}$-estimator and the B-estimator as an estimator of $\sigma^{2}$ or $\sigma$. The J-estimator is better than the $\mathrm{F}$-estimator and is inferior to both $\mathrm{U}$ - and B-estimator for $\sigma^{2}$ and $\sigma$. But the J-estimator is better than those in the interval estimates in many cases. 


\section{Minimum variance unbiased estimator of $\sigma^{2}$}

Since $U$ is a $U$-statistic investigated in detail by Hoeffding(1948) and extended to the two-sample problem by Lehmann(1951), it is easy to show that

$$
\sigma^{2}=\frac{1}{m n}\left\{\zeta_{11}+(m-1) \zeta_{21}+(n-1) \zeta_{12}-(N-1) \zeta_{22}\right\}
$$

where

$$
\begin{aligned}
& \zeta_{11}=\theta=E\left\{u\left(X_{1}-Y_{1}\right)\right\} \\
& \zeta_{21}=E\left\{u\left(X_{1}-Y_{1}\right) u\left(X_{2}-Y_{1}\right)\right\} \\
& \zeta_{12}=E\left\{u\left(X_{1}-Y_{1}\right) u\left(X_{1}-Y_{2}\right)\right\} \\
& \zeta_{22}=\theta^{2}=E\left\{u\left(X_{1}-Y_{1}\right) u\left(X_{2}-Y_{2}\right)\right\}
\end{aligned}
$$

and $N=m+n$. On $U$-statistics, see also Lee(1990).

In order to obtain unbiased estimator of $\sigma^{2}$, we must construct unbiased estimators of $\zeta_{11}, \zeta_{12}, \zeta_{21}$ and $\zeta_{22}$. Define

$$
\begin{gathered}
C_{j}=\sum_{i=1}^{m} u\left(X_{i}-Y_{j}\right), \quad D_{i}=\sum_{j=1}^{n} u\left(X_{i}-Y_{j}\right) \\
C^{2}=\sum_{j=1}^{n} C_{j}^{2}, \quad D^{2}=\sum_{i=1}^{m} D_{i}^{2}, \quad B=\sum_{j=1}^{n} C_{j}=\sum_{i=1}^{m} D_{i}=m n U .
\end{gathered}
$$

Then the minimum variance unbiased estimator of $\zeta_{11}=\theta$ is

$$
\hat{\zeta}_{11}=U=\frac{1}{m n} B .
$$

The minimum variance unbiased estimator of $\zeta_{21}$ is given by a $U$-statistic

$$
\begin{aligned}
\hat{\zeta}_{21} & \left.=\frac{1}{m(m-1) n} \sum_{i \neq i^{\prime}, j} u\left(X_{i}-Y_{j}\right) u\left(X_{i^{\prime}}-Y_{j}\right)\right\} \\
& =\frac{1}{m(m-1) n}\left(C^{2}-B\right)
\end{aligned}
$$

and that of $\zeta_{12}$ is $\hat{\zeta}_{12}=\left(D^{2}-B\right) /\{m n(n-1)\}$. The minimum variance unbiased estimator of $\zeta_{22}$ is given by

$$
\hat{\zeta}_{22}=\widehat{\theta^{2}}=\frac{1}{m(m-1) n(n-1)}\left(B^{2}+B-C^{2}-D^{2}\right) .
$$

Hence, substituting $\hat{\zeta}_{11}, \hat{\zeta}_{21}, \hat{\zeta}_{12}$ and $\hat{\zeta}_{22}$ into (6), an unbiased estimator (U-estimator) of $\sigma^{2}$ is given by

$$
\hat{\sigma}_{U}^{2}=\frac{1}{m(m-1) n(n-1)}\left(-\frac{N-1}{m n} B^{2}-B+C^{2}+D^{2}\right) .
$$




\section{SHIRAHATA}

Since $\hat{\sigma}_{U}^{2}$ is a weighted sum of $U$-statistics, it is also a $U$-statistic with degree $(2,2)$. Though the kernel function depends on $(m, n), \hat{\sigma}_{U}^{2}$ is the minimum variance unbiased estimator of $\sigma^{2}$.

There is a possibility that $\hat{\sigma}_{U}^{2}$ is negative. However, we do not observe the negatives in Monte Carlo experiments in Section 6. The U-estimator is the same with the unbiased estimator given in Sen(1967) where he noted that it is asymptotically normal and that the estimator is a function of ranks.

\section{Bootstrap estimator of $\sigma^{2}$ and Fligner-Policello estimator}

Bootstrap method proposed by Efron $(1979,1982)$ gives good estimators in various fields of statistics. Let us consider bootstrap estimator (B-estimator) of $\sigma^{2}$.

Let $\tilde{X}_{1}, \ldots, \tilde{X}_{m}$ and $\tilde{Y}_{1}, \ldots, \tilde{Y}_{n}$ be samples drawn with replacement from the sets $\left\{X_{1}, \ldots, X_{m}\right\}$ and $\left\{Y_{1}, \ldots, Y_{n}\right\}$, respectively. Put

$$
\tilde{U}=\frac{1}{m n} \sum_{i, j} u\left(\tilde{X}_{i}-\tilde{Y}_{j}\right)
$$

and denote by $E_{C}$ and $\operatorname{var}_{C}$ the conditional mean and the variance given $\left\{X_{1}, \ldots, X_{m}\right.$; $\left.Y_{1}, \ldots, Y_{n}\right\}$. Then, after simple calculations, we have

$$
E_{C}(\tilde{U})=\frac{1}{m n} B
$$

and

$$
E_{C}\left(\tilde{U}^{2}\right)=\frac{1}{m^{2} n^{2}}\left\{\frac{(m-1)(n-1)}{m n} B^{2}+B+\frac{m-1}{m} C^{2}+\frac{n-1}{n} D^{2}\right\} .
$$

From (7) and (8), the B-estimator of $\sigma^{2}$ is given by

$$
\begin{aligned}
\hat{\sigma}_{B}^{2} & =\operatorname{var}_{C}(\tilde{U})=E_{C}\left(\tilde{U}^{2}\right)-\left\{E_{C}(\tilde{U})\right\}^{2} \\
& =\frac{1}{m^{2} n^{2}}\left(-\frac{N-1}{m n} B^{2}+B+\frac{m-1}{m} C^{2}+\frac{n-1}{n} D^{2}\right) .
\end{aligned}
$$

The variance (6) is represented as

$$
\begin{aligned}
\sigma^{2}= & \int G d F-\left(\int G d F\right)^{2}+(m-1)\left\{\int F^{2} d G-\left(\int F d G\right)^{2}\right\} \\
& +(n-1)\left\{\int G^{2} d F-\left(\int G d F\right)^{2}\right\} .
\end{aligned}
$$

Fligner \& Policello(1981) considered an estimator of $\sigma^{2}$ by replacing $F$ and $G$ in (9) with their respective empirical distribution functions. See (3.1) in Fligner \& Policello(1981). By simple calculations, we can find that their estimator (3.1) is equal to $\hat{\sigma}_{B}^{2}$. They also proposed a simple version of (3.1). The simple estimator is

$$
\hat{\sigma}_{F}^{2}=\frac{1}{m^{2} n^{2}}\left(-\frac{N+1}{m n} B^{2}+B+C^{2}+D^{2}\right)
$$


in our notation. We call the estimator (10) F-estimator. A similar consideration is given by Davies \& Phillips(1988) for a modified Mann-Whitney statistic.

\section{Jackknife estimator of $\sigma^{2}$}

Quenouille(1956) and Tukey(1958) proposed jackknife method to estimate variances of statistics. In the two-sample case, we consider the following jackknife estimator.

Compute our $U$-statistic $U_{(i, \cdot)}$ by excluding $X_{i}$ and $U_{(\cdot, j)}$ by excluding $Y_{j}$. The jackknife estimator (J-estimator) of $\sigma^{2}$ is defined as

$$
\hat{\sigma}_{J}^{2}=\frac{m-1}{m} \sum_{i=1}^{m}\left(U_{(i, \cdot)}-U\right)^{2}+\frac{n-1}{n} \sum_{j=1}^{n}\left(U_{(\cdot, j)}-U\right)^{2} .
$$

Using

$$
U_{(i, \cdot)}=\frac{1}{(m-1) n}\left(B-D_{i}\right) \quad \text { and } \quad U_{(\cdot, j)}=\frac{1}{m(n-1)}\left(B-C_{j}\right)
$$

the jackknife estimator is found to be

$$
\hat{\sigma}_{J}^{2}=\frac{1}{m(m-1) n(n-1)}\left(-\frac{N-2}{m n} B^{2}+\frac{m-1}{m} C^{2}+\frac{n-1}{n} D^{2}\right) .
$$

\section{Bias of estimators}

The estimators of the previous sections have the form

$$
\hat{\sigma}^{2}=\frac{1}{m^{2} n^{2}}\left(a B^{2}+b B+c C^{2}+d D^{2}\right) .
$$

Now, consider the bias of $\hat{\sigma}^{2}$. Clearly, $E(B)=m n \zeta_{11}$. By simple calculations, we have

$$
\begin{gathered}
E\left(B^{2}\right)=m n\left\{\zeta_{11}+(m-1) \zeta_{21}+(n-1) \zeta_{12}+(m-1)(n-1) \zeta_{22}\right\} \\
E\left(C^{2}\right)=m n\left\{\zeta_{11}+(m-1) \zeta_{21}\right\}
\end{gathered}
$$

and

$$
E\left(D^{2}\right)=m n\left\{\zeta_{11}+(n-1) \zeta_{12}\right\}
$$

Hence,

$$
\begin{aligned}
\frac{1}{m n} E\left(\hat{\sigma}^{2}\right)= & \frac{1}{m n}\left\{(a+b+c+d) \zeta_{11}+(a+c)(m-1) \zeta_{21}\right. \\
& \left.+(a+d)(n-1) \zeta_{12}+a(m-1)(n-1) \zeta_{22}\right\}
\end{aligned}
$$




\section{SHIRAHATA}

From (6) and (14), we have

$$
\begin{aligned}
E\left(\hat{\sigma}^{2}\right)-\sigma^{2}= & \frac{1}{m n}\left[(a+b+c+d-1) \zeta_{11}+(m-1)(a+c-1) \zeta_{21}\right. \\
& \left.+(n-1)(a+d-1) \zeta_{12}+\{a(m-1)(n-1)+N-1\} \zeta_{22}\right] .
\end{aligned}
$$

From the definition, the bias of $\hat{\sigma}_{U}^{2}$ is zero. Using the above formulae, the bias of $\hat{\sigma}_{B}^{2}, \hat{\sigma}_{F}^{2}$ and $\hat{\sigma}_{J}^{2}$ are found to be

$$
\begin{aligned}
m^{2} n^{2}\left\{E\left(\hat{\sigma}_{B}^{2}\right)-\sigma^{2}\right\}= & (2 m n-2 N+1) \zeta_{11}-(m-1)(N+n-1) \zeta_{21} \\
& -(n-1)(N+m-1) \zeta_{12}+(N-1)^{2} \zeta_{22}, \\
m^{2} n^{2}\left\{E\left(\hat{\sigma}_{F}^{2}\right)-\sigma^{2}\right\}= & (2 m n-N-1) \zeta_{11}-(m-1)(N+1) \zeta_{21} \\
& -(n-1)(N+1) \zeta_{12}+\left(N^{2}-2 m n-1\right) \zeta_{22}
\end{aligned}
$$

and

$$
m n\left\{E\left(\hat{\sigma}_{J}^{2}\right)-\sigma^{2}\right\}=\zeta_{11}-\zeta_{21}-\zeta_{12}+\zeta_{22}
$$

Some examples of the bias of the estimators are given in Table 2 and we feel that, in many cases, the B-estimator underestimates and both the F-estimator and the J-estimator overestimate.

\section{Simulated results}

In this section, let us compare the performance of estimators by computer simulations. We consider five cases given in Table 1 where $N(\cdot, \cdot)$ denotes normal distribution with respective mean and variance and $E(\lambda)$ implies exponential distribution with mean $\lambda$. Sample sizes are selected to be $(m, n)=(20,20),(40,20),(40,40)$ and computations are repeated 100,000 times. The case $(m, n)=(20,20)$ corresponds to the small sample case, the case $(m, n)=(40,40)$ corresponds to the large sample case and the case $(m, n)=(40,20)$ corresponds to the unbalanced case.

Table 1: Simulated Distribution

\begin{tabular}{c|ccccc}
\hline & Case 1 & Case 2 & Case 3 & Case 4 & Case 5 \\
\hline$F$ & $N(0,1)$ & $N(0,4)$ & $N(1,1)$ & $N(1,4)$ & $\operatorname{Exp}(2)$ \\
$G$ & $N(0,1)$ & $N(0,1)$ & $N(0,1)$ & $N(0,1)$ & $\operatorname{Exp}(1)$ \\
\hline
\end{tabular}

Table 2 gives the true variance $\sigma^{2}$, and the true relative bias of $\hat{\sigma}_{U}^{2}, \hat{\sigma}_{B}^{2}, \hat{\sigma}_{F}^{2}$ and $\hat{\sigma}_{J}^{2}$. The relative bias implies the bias divided by the true variance. It is found that $\hat{\sigma}_{B}^{2}$ has a tendency to underestimate and both $\hat{\sigma}_{F}^{2}$ and $\hat{\sigma}_{J}^{2}$ overestimate $\sigma^{2}$.

Table 3 gives the simulated mean squared error where the rows of $\hat{\sigma}_{U}^{2}, \hat{\sigma}_{B}^{2}, \hat{\sigma}_{F}^{2}$ and $\hat{\sigma}_{J}^{2}$ correspond to the respective estimates of $\sigma^{2}$ and the rows of $\hat{\sigma}_{U}, \hat{\sigma}_{B}, \hat{\sigma}_{F}$ and $\hat{\sigma}_{J}$ correspond to the respective estimates of $\sigma$. The mean squared errors in Table 3 are computed from the relative values, i.e., simulated value divided by the true value. 
Estimate of Variance of Wilcoxon-Mann-Whitney Statistic

Table 2: True variance and true relative bias $\left(\times 10^{3}\right)$

\begin{tabular}{c|c|c|cccc}
\hline Case & Sample sizes & $\sigma^{2}$ & $\hat{\sigma}_{U}^{2}$ & $\hat{\sigma}_{B}^{2}$ & $\hat{\sigma}_{F}^{2}$ & $\hat{\sigma}_{J}^{2}$ \\
\hline 1 & 20,20 & 8.54 & 0 & -4.82 & 43.8 & 24.4 \\
& 40,20 & 6.35 & 0 & -10.6 & 22.1 & 16.4 \\
& 40,40 & 4.22 & 0 & -1.23 & 23.4 & 12.3 \\
\hline 2 & 20,20 & 9.16 & 0 & -14.4 & 34.0 & 19.2 \\
& 40,20 & 8.27 & 0 & -27.4 & 39.9 & 10.6 \\
& 40,40 & 4.53 & 0 & -6.32 & 18.3 & 9.70 \\
\hline 3 & 20,20 & 5.75 & 0 & 7.05 & 56.0 & 30.8 \\
& 40,20 & 4.27 & 0 & -2.40 & 30.5 & 20.7 \\
& 40,40 & 2.83 & 0 & 5.12 & 29.9 & 15.6 \\
\hline 4 & 20,20 & 7.89 & 0 & -11.5 & 37.0 & 20.8 \\
& 40,20 & 7.14 & 0 & -25.8 & 43.1 & 11.5 \\
& 40,40 & 3.91 & 0 & -4.77 & 19.9 & 10.5 \\
\hline 5 & 20,20 & 7.42 & 0 & -1.43 & 47.3 & 26.2 \\
& 40,20 & 5.93 & 0 & -13.0 & 32.7 & 16.4 \\
& 40,40 & 3.66 & 0 & -0.58 & 25.3 & 13.3 \\
\hline
\end{tabular}

From Table 3, we can find that the B-estimators $\hat{\sigma}_{B}^{2}$ and $\hat{\sigma}_{B}$ are the best for $\sigma^{2}$ and $\sigma$, respectively, in the sense of mean squared error. The U-estimators $\hat{\sigma}_{U}^{2}$ and $\hat{\sigma}_{U}$ are the second best. However, the mean squared errors of $\hat{\sigma}_{B}^{2}$ and $\hat{\sigma}_{B}$ are not surprisingly small so that we should use $\hat{\sigma}_{U}^{2}$ and $\hat{\sigma}_{U}$ if we attach importance in the unbiasedness. The J-estimators and F-estimators are not efficient. However, $\hat{\sigma}_{J}$ has nearly equal mean squared errors to that of $\hat{\sigma}_{U}$.

Table 3: Simulated results on mean squared error $\left(\times 10^{3}\right)$

\begin{tabular}{c|c|cccc|cccc}
\hline Case & $m, n$ & $\hat{\sigma}_{U}^{2}$ & $\hat{\sigma}_{B}^{2}$ & $\hat{\sigma}_{F}^{2}$ & $\hat{\sigma}_{J}^{2}$ & $\hat{\sigma}_{U}$ & $\hat{\sigma}_{B}$ & $\hat{\sigma}_{F}$ & $\hat{\sigma}_{J}$ \\
\hline 1 & 20,20 & 4.35 & 3.95 & 6.22 & 4.92 & 1.17 & 1.07 & 1.56 & 1.27 \\
& 40,20 & 9.99 & 8.83 & 9.38 & 9.38 & 2.49 & 2.24 & 2.27 & 2.41 \\
& 40,40 & 1.05 & 1.00 & 1.59 & 1.20 & 0.27 & 0.26 & 0.40 & 0.30 \\
\hline 2 & 20,20 & 7.65 & 6.59 & 8.20 & 7.71 & 1.99 & 1.76 & 2.04 & 1.96 \\
& 40,20 & 2.20 & 2.08 & 3.74 & 2.24 & 0.56 & 0.53 & 0.91 & 0.58 \\
& 40,40 & 2.66 & 2.42 & 2.84 & 2.67 & 0.67 & 0.62 & 0.70 & 0.67 \\
\hline 3 & 20,20 & 76.5 & 72.5 & 83.0 & 79.4 & 21.9 & 20.4 & 21.7 & 21.6 \\
& 40,20 & 70.6 & 65.9 & 70.6 & 82.7 & 19.2 & 17.9 & 18.3 & 26.1 \\
& 40,40 & 37.5 & 36.6 & 39.3 & 38.2 & 10.0 & 9.69 & 10.0 & 9.96 \\
\hline 4 & 20,20 & 36.1 & 32.9 & 37.4 & 36.6 & 10.3 & 9.50 & 10.0 & 10.1 \\
& 40,20 & 18.1 & 17.4 & 20.6 & 23.7 & 4.87 & 4.67 & 5.18 & 6.82 \\
& 40,40 & 17.0 & 16.2 & 17.4 & 17.1 & 4.51 & 4.33 & 4.48 & 4.47 \\
\hline 5 & 20,20 & 30.3 & 28.2 & 33.3 & 31.5 & 8.59 & 7.99 & 8.74 & 8.55 \\
& 40,20 & 25.6 & 23.8 & 26.2 & 30.2 & 6.79 & 6.36 & 6.64 & 8.67 \\
& 40,40 & 14.1 & 13.7 & 14.9 & 14.4 & 3.75 & 3.62 & 3.81 & 3.74 \\
\hline
\end{tabular}

We also computed empirical relative bias of the estimators. Though the results are not listed in the tables, we found that the simulated bias of $\hat{\sigma}_{B}^{2}, \hat{\sigma}_{F}^{2}$ and $\hat{\sigma}_{J}^{2}$ are close to the true 


\section{SHIRAHATA}

ones in Table 2. We also found that the bias of $\hat{\sigma}_{U}$ is small, $\hat{\sigma}_{B}$ underestimates in many cases and $\hat{\sigma}_{F}$ and $\hat{\sigma}_{J}$ overestimate.

Table 4: Simulated results of interval estimate

\begin{tabular}{|c|c|c|c|c|c|c|c|c|c|}
\hline & \multicolumn{3}{|c|}{ Case 1} & \multicolumn{3}{|c|}{ Case 2} & \multicolumn{3}{|c|}{ Case 3} \\
\hline & 20,20 & 40,20 & 40,40 & 20,20 & 40,20 & 40,40 & 20,20 & 40,20 & 40,40 \\
\hline$\overline{U, N}$ & 93537 & 93933 & 94252 & 93563 & 94269 & 94352 & 92446 & 92879 & 93683 \\
\hline$U, t$ & 94275 & 94419 & 94584 & 94294 & 94728 & 94668 & 93107 & 93311 & 94031 \\
\hline$U, N, C$ & 93697 & 94042 & 94307 & 93718 & 94383 & 94402 & 92633 & 92995 & 93759 \\
\hline$U, t, C$ & 94432 & 94520 & 94645 & 94443 & 94822 & 94709 & 93293 & 93429 & 94098 \\
\hline$B, N$ & 93517 & 93821 & 94254 & 93439 & 94263 & 94293 & 92634 & 92940 & 93785 \\
\hline$B, t$ & 94256 & 94327 & 94581 & 94162 & 94717 & 94626 & 93313 & 93365 & 94113 \\
\hline$B, N, C$ & 93684 & 93930 & 94302 & 93597 & 94381 & 94346 & 92826 & 93044 & 93840 \\
\hline$B, t, C$ & 94414 & 94415 & 94635 & 94330 & 94820 & 94665 & 93508 & 93493 & 94189 \\
\hline$F, N$ & 94057 & 94218 & 94505 & 93979 & 94716 & 94556 & 93099 & 93293 & 94044 \\
\hline$F, t$ & 94756 & 94736 & 94858 & 94664 & 95166 & 94860 & 93764 & 93732 & 94359 \\
\hline$F, N, C$ & 94239 & 94321 & 94562 & 94134 & 94806 & 94604 & 93329 & 93403 & 94114 \\
\hline$F, t, C$ & 94894 & 94815 & 94909 & 94816 & 95261 & 94896 & 93937 & 93836 & 94427 \\
\hline$J, N$ & 93832 & 93679 & 94384 & 93798 & 94177 & 94455 & 92800 & 91335 & 93877 \\
\hline$J, t$ & 94566 & 94173 & 94734 & 94507 & 94634 & 94765 & 93474 & 91841 & 94209 \\
\hline$J, N, C$ & 93979 & 93780 & 94438 & 93955 & 94299 & 94504 & 92998 & 91480 & 93948 \\
\hline \multirow[t]{3}{*}{$J, t, C$} & 94701 & 94264 & 94786 & 94646 & 94730 & 94816 & 93667 & 91980 & 94281 \\
\hline & \multicolumn{3}{|c|}{ Case 4} & \multicolumn{3}{|c|}{ Case 5} & & & \\
\hline & 20,20 & 40,20 & 40,40 & 20,20 & 40,20 & 40,40 & & & \\
\hline$\overline{U, N}$ & 92961 & 93887 & 93997 & 93070 & 93670 & 94159 & & & \\
\hline$U, t$ & 93632 & 94354 & 94338 & 93827 & 94164 & 94489 & & & \\
\hline$U, N, C$ & 93141 & 94006 & 94053 & 93262 & 93803 & 94212 & & & \\
\hline$U, t, C$ & 93802 & 94455 & 94414 & 94002 & 94253 & 94547 & & & \\
\hline$B, N$ & 92910 & 93928 & 93963 & 93137 & 93694 & 94184 & & & \\
\hline$B, t$ & 93590 & 94391 & 94303 & 93875 & 94171 & 94514 & & & \\
\hline$B, N, C$ & 93081 & 94035 & 94021 & 93316 & 93805 & 94235 & & & \\
\hline$B, t, C$ & 93760 & 94510. & 94361 & 94058 & 94275 & 94565 & & & \\
\hline $\bar{F}, N$ & 93427 & 94379 & 94241 & 93689 & 94109 & 94444 & & & \\
\hline$F, t$ & 94098 & 94830 & 94593 & 94386 & 94543 & 94782 & & & \\
\hline$F, N, C$ & 93575 & 94493 & 94287 & 93854 & 94207 & 94500 & & & \\
\hline$F, t, C$ & 94250 & 94947 & 94649 & 94536 & 94627 & 94840 & & & \\
\hline$J, N$ & 93217 & 93197 & 94119 & 93406 & 93003 & 94301 & & & \\
\hline$J, t$ & 93899 & 93680 & 94484 & 94138 & 93459 & 94633 & & & \\
\hline$J, N, C$ & 93394 & 93328 & 94185 & 93595 & 93120 & 94360 & & & \\
\hline$J, t, C$ & 94064 & 93799 & 94550 & 94317 & 93573 & 94693 & & & \\
\hline
\end{tabular}

We further investigate the interval estimates of $\theta$ based on $U$ standardized by our 
estimators of $\sigma$. Sixteen interval estimators are considered. They are of the form

$$
U-\frac{a}{m n}-c \hat{\sigma}<\theta<U+\frac{a}{m n}+c \hat{\sigma}
$$

where the estimator $\hat{\sigma}$ is one of $\hat{\sigma}_{U}, \hat{\sigma}_{B}, \hat{\sigma}_{F}$ and $\hat{\sigma}_{J}, a=0$ or $a=\frac{1}{2}$ (finite correction) and $c$ is the upper $\alpha / 2$ point of standardized normal distribution or that of $t$-distribution with degrees of freedom $m+n-2$. We also simulated iterated bootstrap estimator. However, the performance of the estimator is poor and hence the results are omitted.

The simulated results repeated 100,000 times on interval estimates are given in Table 4 where the entries are the number of times which the interval estimates with $95 \%$ confidence coefficient included true $\theta$ and it is better when the entries are close to 95,000. In Table $4, U, B, F$ and $J$ corresponds to the U-,B-,F- and J-estimator, respectively. The $N$ and $t$ correspond to the normal and t-approximation, respectively. Further, $C$ implies that we used the finite correction. No method is the best at all times. However, it can be said that the estimators with finite corrected $t$-approximation is efficient in many cases. The finite correction is not very effective whereas the $t$-approximation is very effective. Among four estimators of $\sigma$, the F-estimator gives the most accurate sample significances. The accuracy of the sample significances of the J-estimator are the second best in many cases. These phenomena are, we think, due to the fact that the $\mathrm{U}$ - and the B-estimator underestimate $\sigma$ with large probability and hence the interval is too narrow. Hence, though $\hat{\sigma}_{F}$ is not efficient as a point estimator of $\sigma$, the estimator is the most useful in the interval estimate of $\theta$.

\section{Acknowledgement}

The author thanks to the referees for their careful reading the original manuscript and helpful comments.

\section{REFERENCES}

Davies, P. \& Phillips, A. J. (1988). Nonparametric tests of population differences and estimation of the probability of misidentification with unidentified paired data. Biometrika 75, 753-760.

Efron, B. (1979). Bootstrap methods: another look at the jackknife. Ann. Statist. 7, 1-26.

Efron, B. (1982). The Jackknife, the Bootstrap and Other Resampling Plans. Philadelphia: SIAM.

Fligner, M. A. \& Policello, II. G. E. (1981). Robust rank procedures for the Behrens-Fisher problem. J. Am. Statist. Assoc. 76, 162-168.

Hodges, Jr. J. L. \& Lehmann, E. L. (1963). Estimates of location based on rank tests. Ann. Math. Statist. 34, 598-611. 


\section{SHIRAHATA}

Hoeffding, W. (1948). A class of statistics with asymptotically normal distribution. Ann. Math. Statist. 19, 293-325.

Lee, A. (1990). U-statistics. M.Dekker, New York.

Lehmann, E. L. (1951). Consistency and unbiasedness of certain nonparametric tests. Ann. Math. Statist. 22, 165-179.

Lehamnn, E. L. (1963). Nonparametric confidence intervals for a shift parameter. Ann. Math. Statist. 34, 1507-1512.

Mann, H. B. \& Whitney, D. R. (1947). On a test of whether one of two random variables is stochastically larger than the other. Ann. Math. Statist. 18, 50-60.

Milton, R. C. (1964). An extended table of critical values for the Mann-Whitney (Wilcoxon) two-sample statistic. J. Am: Statist. Assoc. 59, 925-934.

Quenouille, M. (1956). Notes on bias in estimation. Biometrika 43, 353-360.

Sakamoto, Y. \& Shirahata, S. (1992). Estimate of the variance of $U$-statistics. Communi.Statist.21, Commun.Statisy.,Theo.Method, 21, 2969-2981.

Sen, P. K. (1967). A note on asymptotically distribution-free confidence bounds for $P\{X<$ $Y$ \}, based on two independent samples. Sankhya A 29, 95-102.

Tukey, J. (1958). Bias and confidence in not quite large samples. Ann. Math. Statist. 29, 614.

Wilcoxon, F. (1945). Individual comparisons by ranking methods. Biometrics Bull. 1, 80-83.

(Received May 1993; Revised July 1993) 